\title{
Bridging the Left - Right Political Divide with Artificial Intelligence and Collective Intelligence
}

\author{
Andy E. Williams ${ }^{1, *}$ \\ ${ }^{1}$ Nobeah Foundation, Nairobi, Kenya \\ Correspondence*: \\ Corresponding Author \\ awilliams@nobeahfoundation.org
}

\begin{abstract}
A model of cognition suggests that the left vs right political debate is unsolvable. However the same model also suggests that a form of collective cognition (General Collective Intelligence or $\mathrm{GCl}$ ) can allow education, health care, or other government services to be customized to the individual, so that individuals can choose services anywhere along the spectrum from socialized services if they desire, or private services if they desire, thereby removing any political stalemate where it might prevent any progress. Whatever services groups of individuals choose, $\mathrm{GCl}$ can significantly increase the quality of outcomes achievable through either socialized or private services today, in part through using information regarding the fitness of any services deployed, to improve the fitness of all services that might be deployed. The emerging field of General Collective Intelligence $(\mathrm{GCl})$ explores how platforms might increase the general problem-solving ability (intelligence) of groups so that it is significantly higher than that of any individual. Where Collective Intelligence $(\mathrm{Cl})$ must find the optimal solution to a problem or group of problems, having general problem-solving ability, a $\mathrm{GCl}$ must also have the capacity to find the optimal problem to solve. In the case of political discussions, $\mathrm{GCI}$ must have the ability to re-frame political discourse from being focused on questions that have not proved resolvable, such as whether or not left leaning or right leaning political opinions are in general more "right" or "wrong". Instead $\mathrm{GCl}$ must have the ability to refocus discussions, including on how to objectively determine whether a left or right bias optimizes outcomes in a specific context, and why. This paper explores the conjecture that determining whether a left leaning or right leaning cognitive bias is "optimal" (i.e. "true) based on any $\mathrm{Cl}$ or other aggregate of individual reasoning that is not $\mathrm{GCl}$, cannot reliably converge on "truth" because each individual cognitive bias leads to evaluating truth according to different reasoning types (type 1 or type 2) that might give conflicting answers to the same problem. However, through using functional modeling to create the capacity to represent all possible reasoning processes, and through using functional modeling to represent the domains in conceptual space in which each reasoning process is optimal, it is possible to systematically categorize an unlimited number of collective reasoning processes and the contexts in which execution of those reasoning processes with a right leaning or left leaning bias is optimal for the group. By designing $\mathrm{GCl}$ algorithms to incorporate each bias in its optimal context, a $\mathrm{GCl}$
\end{abstract}


can allow individuals to participate in collective reasoning despite their biases, while collective reasoning might still converge on "truth" in terms of functioning to optimize collective outcomes. And by deploying intelligent agents incorporating some subset of AGI to interact on the individual's behalf at significantly higher speed and scale, collective reasoning might gain the capacity to consider all reasoning and all "facts" available to any individual in the group, in order to converge on that truth while significantly increasing outcomes.

Keywords: Artificial General Intelligence, General Collective Intelligence, cognitive computing, collective cognitive computing, cognitive bias, political bias, e-government, health care, education

\section{INTRODUCTION}

A recently developed Human-Centric Functional Modeling approach (HCFM) (Williams, 20201) models systems in terms of the minimal set of functions through which the system can change it's state. These states form the "functional state space" through which the system moves. By identifying a set of functions that can be observed within innate human awareness (human-centric) as opposed to functions which assume any particular theory of implementation, simple models of complex systems can be defined even where the mechanisms by which those functions are implemented are currently unknown. Because understanding any problem regarding such a system becomes a problem of understanding the processes by which the system can navigate from one state to another, and because a human-centric set of functions can more readily be navigated within the space of human cognition, this HCFM approach can be used to reliably "compute" the solutions to far more complex problems than currently achievable otherwise.

Using this HCFM approach, a Functional Modeling Framework (FMF) (Williams, 2020h) has been recently defined to model the human system, including cognition. The FMF represents the cognitive system in terms of a cognitive awareness process that uses reasoning processes to navigate a space of concepts (a "conceptual space"). In this framework, the cognitive awareness process detects where the cognitive system is in conceptual space, and chooses which reasoning process to execute next in a way that maintains the fitness of the system to execute all its functions (the cognitive "well-being) within a stable range. By defining the boundary conditions of stability, by defining the functions required to maintain stability, and by defining a set of functions by which any possible conceptual space can be navigated, a model believed to represent all the functions of cognition has been developed.

This model represents both of the two types of reasoning that human beings are observed to have, namely type 1 (intuitive or "fast") reasoning, and type 2 (rational methodical or "slow") reasoning. Any problem that is computable can potentially be solved by some series of well-defined steps (Sipser, 1996). Any such set of well-defined steps can be represented using type 2 reasoning. Type 2 reasoning in this way is analogous to computing the result of an "equation". It's important to note that the label "rational methodical reasoning" does not equate to "rational" or "correct" since it may be evaluated incorrectly. Furthermore, if an appropriate equation does not exist, or if the inputs or constants required to evaluate that equation are not available, this reasoning approach cannot be used at all. Type 1 reasoning on the other hand is equivalent to determining the solution from observation of patterns of solutions in the past. With type 1 reasoning a method for computing the solution need not even exist. For this reason, type 1 reasoning can manage non-computable problems.

In representing all of what cognition can do, this is believed to be the first model of cognition with the potential for human-like general problem-solving ability (intelligence). Because it is a functional model independent of implementation, it has also been used to define what is believed to be the first model 
of artificial cognition with the potential for human-like intelligence (Artificial General Intelligence or AGI (Williams, 2020j)). Being independent of implementation, this model has also been used to define a model of collective cognition (General Collective Intelligence or GCI (Williams, 2019)). Because these HCFM and FMF approaches are so new, all the models of AGI and GCI developed using these approaches have originated from a single author, the author of this current paper. However, though for this reason all references to these models refer to the work of this author, the concept of a general collective intelligence factor (Woolley et al. 2010) and the concept of achieving a collective "super intelligence" (Malone, 2018) have been pioneered by others.

\section{DEFINING GENERAL COLLECTIVE INTELLIGENCE}

General Collective Intelligence or GCI has been defined to be a platform that organizes groups to have the potential for significantly greater general problem-solving ability than that of any individual in the group (Williams, 2020k). Where Collective Intelligence (CI) must find the optimal solution to a problem or group of problems, having general problem-solving ability, a GCI must also have the capacity to find the optimal problem to solve. In order to so, GCI solves the more general problem of collective well-being.

Recent work (Williams, 2020i) has proposed a mechanism for intelligence and how intelligence might be quantified. This model has calculated the potential increase in intelligence that is possible with GCI as being exponential in comparison with the intelligence of any individual. According to this model, an exponential increase in general problem-solving ability has not been possible before at any time in the history of civilization, and cannot be again until the implementation of either AGI or a second order GCI. An exponential increase in general problem-solving ability potentially represents an exponential ability to achieve impact on the external environment. Other work has assessed the potential implications of GCI and this impact in a wide range of areas, including the Sustainable Development Goals (SDGs) (Williams, 2020b). In short, GCI is essentially a system for maximizing collective outcomes, but since by design a GCI is required to be a dynamically stable system with respect to available resources, and since any outcome in general might be required to achieve or restore stability, GCI must must also maximize the degree to which it can achieve collective outcomes per unit of resources.

According to the model of GCI, without such a system all current decision systems, whether autocratic rule or democratic voting, contain features or bugs that align decision-making with the interests of some subset of individuals rather than with the collective well-being (Williams, 2020a), (Williams, 2020e). This holds both for decision-making on the political left in the US, as well as on the political right, and is especially apparent during times of crisis that draw attention to flaws in decision-making, such as the current COVID-19 pandemic (Williams, 2020g).

\section{USING GENERAL COLLECTIVE INTELLIGENCE TO RE-FRAME AND RE-FOCUS POLITICAL DISCOURSE}

In the case of political discussions, having the general problem-solving ability to choose the optimal problem to solve, a GCI must have the ability to re-frame political discourse from being focused on questions that have not proved resolvable (Rapaport, 1982), such as whether or not left leaning or right leaning political opinions are in general more "right" or "wrong" (Baron and Jost, 2019), (Washburn and Skitka, 2018), (LUTTBEG and GANT, 1985). Access to information does not appear to resolve such questions, as biases lead individuals to remain in their own echo chambers (Bowen et al., 2020). Conservatives often describe the "liberal bias" of the great many mainstream media outlets that fall outside the conservative narrative (Groseclose, 2012). Liberals on the other hand often describe media that falls outside the liberal narrative as being "fake news" that the public should be protected against through 
censorship (Morgan, 2018) - (Zhuk et al., 2018). In the face of this increasing polarization, where media might have shaped public opinion in the past (Bernhardt et al., 2008), public confidence in news media in general has eroded to an all time low (Lewis, 2020).

While there are certainly people on each side who are more focused on conflict rather than collective well-being, in this left vs right political divide, each side might be predisposed to believe the other side is not concerned with collective well-being at all. Those conservatives focused on collective well-being might be predisposed to believe that no one is more incentivized to act in an individual citizen's interest than the individual citizen themselves (Béland and de Chantal, 2004), and that maximizing public well-being can only be achieved through minimizing any government infringement on those rights and freedoms. Rather than seeing liberals as working for the common good, conservatives might be more likely to perceive liberals as wanting to erode the rights and freedoms that enable individuals to take responsibility for choosing values that allow them to achieve their well-being, in the misguided hope that "big daddy government" will achieve their well-being for them (Marshall, 1996). Liberals focused on collective well-being on the other hand might be predisposed to believe that only governments have the resources to help ensure the well-being of any individual (Yankelovich, 2015), and that maximizing collective well-being can only be achieved effectively through the impact of government programs. Rather than seeing conservatives as working for the common good, liberals might be more likely to perceive conservatives as not caring about the well-being of others, or being openly hostile to others (e.g. racist) (Kidder, 2018). Perceiving the other side as not being concerned with collective-well being is likely equivalent to seeing the other side as "morally wrong" or even "evil". Discussion requires the ability to listen to another point of view. And listening requires some degree of empathy (M.D., 1981). There can be no empathy, and therefore no discussion with those perceived to be evil (Saka and Cohen-Louck, 2014a)

If it is true that intuitive reasoning processes detect solutions to non-computable problems, then finding rational methodical reasoning processes that map to those conclusions is an unsolvable problem. The arguments presented in this paper apply to liberals and conservatives in the US. The definition of liberal or conservative in other countries might be very different. Any broad assumption that US liberals use only intuitive reasoning or that US conservatives use only rational methodical reasoning must be incorrect according to this model of cognition. According to this model, each individual uses both, with rational methodical reasoning being used when there is a well-defined reasoning process (i.e. an equation) by which a conclusion can be drawn, and intuitive reasoning being used when the issue is too complex and attempting to reduce it to any such equation leaves out important considerations and therefore is likely to result in unjust or even offensive conclusions. Though it would be incorrect to assume any demographic engages in only type 1 or type 2 reasoning, this paper proposes that different individuals might tend to use type 1 reasoning in the same topics in which other individuals might tend to use type 2 reasoning. And that these tendencies are not equally distributed by demographic, by left or right political bias, and potentially by any number of other categories by which individuals might be grouped. If mapping type 1 reasoning to type 2 reasoning is a non-computable problem, then if intuitive or type 1 reasoning on a given topic is associated with leftism, and if rational methodical reasoning on a given topic is associated with rightism, attempting to find a solution to the problem of convincing individuals with leftist reasoning of the correctness of right leaning reasoning, or of convincing individuals with right-leaning reasoning of the correctness of leftist reasoning, is also a non-computable problem.

Rather than unproductively addressing non-computable problems (Saka and Cohen-Louck, 2014b), GCI must manage unsolvability through the ability to refocus discussions on questions that might lead to new insights regarding the relationship between decision-making mechanisms and general problem-solving, 
where those insights might significantly increase impact on the targeted problems. An example of such insight is how to objectively determine whether a left or right bias optimizes outcomes in a specific context, and why.

\section{IMPLEMENTING A GCI TO OPTIMIZE COLLECTIVE DECISION-MAKING IN THE FACE OF EACH INDIVIDUAL'S POLITICAL BIASES}

From the functional modeling perspective, "truth" is whatever functions to achieve an outcome. For example, if whatever rock one throws up always comes down, then gravity is the truth. If sometimes it simply floats away, then gravity is only part of the truth. Through using functional modeling to create the capacity to represent all possible reasoning processes, and through using functional modeling to represent the domains in conceptual space in which each reasoning process is optimal, it is possible to systematically categorize an unlimited number of collective reasoning processes and the contexts in which execution of those reasoning processes with a right leaning or left leaning bias is optimal for the group. By designing GCI algorithms to incorporate each bias in its optimal context, a GCI can allow individuals to participate in collective reasoning with their biases, while collective reasoning might still converge on "truth" in terms of functioning to optimize collective outcomes. And by having intelligent agents incorporating some subset of AGI to interact on the individual's behalf at significantly higher speed and scale, collective reasoning might gain the capacity to consider all reasoning and all "facts" available to any individual in the group, in order to converge on that truth.

Anecdotally, liberals might be predisposed to believe that conservatives don't care about other people, or that conservatives even wish other specific groups harm (e.g. are rascist). Whether or not conservatives care about other people might not be likely to bar the implemention of GCI based services that not only allow liberals to make liberal choices, but that also allow conservatives to make conservative choices. The issue may be more likely that any liberal predisposition to restrict collective choices to those they believe are consistent with what from the left's perspective is the common good, means that the freedom for conservatives to make conservative choices can only occur either if it is outside of the ability of liberals to restrict, or if that freedom is phrased in a way that liberals can agree with. In a GCI based system conservatives will be setting policies for people who have the same beliefs as themselves. Even if liberals are predisposed to believe that conservatives care only about themselves, by extension conservatives must care about all these others like themselves, potentially providing a counter to arguments that would restrict conservative choices.

\section{DECENTRALIZING COLLECTIVE DECISION-MAKING THROUGH GCI}

According to this model of collective cognition (GCI), in order to remove the bugs or features in current group decision-making so that truly decentralized collective decision-making is possible, "collective reasoning" must be possible (Williams, 2020e). According to the models of individual cognition and collective cognition described here, where the individual cognitive awareness process navigates individual reasoning in order to maintain cognitive fitness ("well-being") within a stable range, the collective cognitive awareness process navigates collective reasoning in order to maintain collective cognitive well-being within a stable range. Just like the intelligence of one individual can be distinguished from the intelligence of another individual in this model by the fact that the function of any individual's intelligence is fundamentally aligned with maintaining their cognitive well-being, the intelligence of the collective then differs from the intelligence of any particular individual in the collective in being fundamentally aligned with the collective well-being (Williams, 2019). 
In order to be reliably aligned with collective well-being, that is, in order to be able to collectively reason, a GCI must have certain functional elements (Williams, 2020k) that eliminate the barriers to finding an incorporating the best ideas and information available in the group, and that enable the group to reliably converge on optimal conclusions.

Either individual or collective reasoning can be used to define an intervention by which the group might optimize some collective outcome. If the claims that GCI can potentially exponentially increase collective outcomes (Williams, 2020i) (depending on the size of the group) are true, then it might be expected that using collective reasoning to define interventions is vastly more preferable. Since GCI has not yet been implemented, this potentially makes implementing GCI important to all collective interventions. And whether in health care, education, the legal system, the justice system, or other area, every interaction with an individual or group that has a targeted outcome is an intervention. The collective interventions we have chosen, or that have been imposed on us, are our systems of governance.

Since governance is preferably the manifestation of a collective reasoning process, the potential of GCI to decentralize collective reasoning so that allows individuals to participate in collective reasoning with their biases, while still converging on optimal collective outcomes, implies that through GCI, systems of governance (reasoning processes providing governance) can be decentralized to the individual in a way that enables individuals to choose the style of governance they believe in, while collectively evolving according to those individual choices in a way that naturally converges on optimal collective well-being, so that decision-making to select systems of governance is no longer a cause for division.

In such a system left-leaning individuals might select systems of governance that cater to their sense of inclusion and benefits for all, and right leaning individuals might select systems of governance that cater to their sense of responsibility and exclusion for those who refuse to assume that responsibility. With GCI, individuals who assume sub-optimal strategies might be incentivized or coerced (promised the carrot and threatened with the stick) into choosing more optimal ones in a way that evolves and converges towards optimal collective outcomes. Individuals who have benefited through choosing optimal strategies might be incentivized or coerced in the same way into taking responsibility for or including those who have taken sub-optimal strategies.

The concept of an AGI and GCI based system coming up with "carrots and sticks" might appear to verge on dictatorial. What if those carrots or sticks aren't always moral or ethical approaches? The answer is that without taking a functional modeling approach, what is moral or ethical is relative and subjective. Subjective problems might be unsolvable. For example, deciding whether gravity is moral or ethical is unsolvable. However, defining truth as whatever functions to achieve an outcome (i.e. defining gravity as the function governing which way objects go when they run out of momentum after being thrown up) is reliably solvable.

Given that a GCI must provide "carrots and sticks", exactly how the individual is free to choose in a system of GCI might not be clear. As an example, one individual might choose a health care system that encourages them to drink and smoke. A GCI might combine them with other people who don't want to be told not to drink and smoke to find cooperative synergies through which GCI might maximize health outcomes for that group. A GCI might also show that individual that other groups achieve much better outcomes by not drinking or smoking. And a GCI might organize the larger community to incentivize and pressure other groups to incentivize and pressure that individual to change to one of those other groups, to avoid the instability that comes with any inequality of outcomes that leads to one group coming into conflict with another. 
What might such a decentralized system of governance look like? Viewing governance as a service in some category of need (e.g. health care, housing, employment, etc.), then each service consists of a set of processes, each of which trace a path through the collective conceptual space. Depending on factors such as complexity, each such path can be executed by a given set of individuals with a given level of fitness as determined by the resources consumed per volume of outcomes. For each service, there are inputs which have a cost, and a volume of collective outcomes that have a value. There might be tens, thousands, millions, or even billions or more patterns of collectively intelligent cooperation through which cooperation might be scaled to reduce costs, increase outcomes, or to increase capacity to navigate more complex processes to further reduce costs or increase outcomes. The purpose of GCI is to enable individuals to make choices to maximize their outcomes, while considering a vastly greater amount of information than any individual can navigate (e.g. these patterns of collectively intelligent cooperation) to reliably converge collectively on choices that maximize collective outcomes.

The Functional Modeling Framework used to derive this model of GCI represents all living systems as having a global stability in fitness space throughout all regions of functional state space, despite being locally unstable in that functional state space. This dynamical stability means living systems can scale in whatever way that is required to be self-sustaining. If plankton feeding in both tropical and antarctic seas requires large size, the organism might become a cooperation between a larger and larger number of cells until it grows to the size of a whale. If gathering seeds one by one requires small size, the organism might become a cooperation between a fewer and fewer number of cells until it shrinks to the size of a mouse.

The value created by this collectively intelligent cooperation in a GCI can reliably be scaled to the point at which it can support the cost of whatever services is sustainable (dynamically stable). The optimal implementation of the service for each individual might be achieved by scaling the cooperation optimally depending on the size of the group. Since collective reasoning might take any path through the collective conceptual space where that path is optimal, and since any given individual or group without GCI is restricted to an exponentially smaller conceptual space, services not defined using collective reasoning are overwhelmingly likely to be sub-optimal. They are also not required to be dynamically stable, the cost of sub-optimal services that are not dynamically stable cannot be ensured to be sustainably supported. In a collective intelligence based service in which individuals are free to choose the implementation of services they believe to be optimal, being dynamically stable, the difference in cost vs benefit of the patterns of collectively intelligent cooperation that are chosen accrue in a way that most reliably causes choices to evolve towards better ones. In a system of GCI any component can be replaced with one that is more fit. This applies here as well. The algorithm by which choices evolve to better ones is managed by a GCI process that can replace it in any given context if another algorithm is found to be more fit in that context.

For example, consider a GCI based educational system. In a GCI based educational system, individuals would be free to select the educational system that fits their political views. Those with left leaning political views would be free to choose socialized (public) education, and those with right leaning political views would be free to choose private education. In both instances, GCI is designed to scale the value of cooperation within the sub-group choosing that option until that value allowed access to significantly more affordable education, or significantly improved education at the same cost, than any socialized educational system or private educational system is capable of today without GCI.

Participation in the potential chains of cooperation through which value might be created for the student would define a preferred educational path but would not confine the student to one. If an individual chooses to get educated in a field in which GCI has determined they have the aptitude to succeed, and in which there are instructors and programs to train them, and in which there are jobs available, with whatever margin 
of error the sub-community agrees to, then the community might reliably ensure any education required to achieve that goal is covered, and furthermore might deploy value chains of cooperating businesses if necessary to reliably ensure there is a job waiting for that individual. If the individual chooses to get educated in another field that is outside the margin of error the community agrees to, then the individual might simply join a different sub-community. In the case that there are no sub-communities that share the same values, then the system reverts to the current situation in some countries in which the individual provides for their own choices, such as getting a loan to finance their own education. In essence, individuals select optimal options according to their choice of strategy. Their collectively beneficial or negative outcomes can be paid for by the cooperation among the sub-group in the collective sharing that strategy, or revert to the current situation in which they pay for their personally beneficial or negative outcomes themselves. In other words, each individual would decide what service delivery policies they want to abide by. GCI would automatically opt them in to the community that agrees to those policies in order to scale value to a large enough degree to pay for those policies.

In GCI based health care services, the same general pattern applies. Currently, in the world without GCI every health care system makes tradeoffs in service levels rather than optimizing across all outcomes. For example, every system explicitly or implicitly puts a cost limit on individual human life. While the budget that it is possible to expend in trying different treatments is potentially unlimited, this cost limit determines whether tens of millions can be spent on treatment for one individual with a rare or difficult illness, or whether that budget is spread over thousands of patients with more common ailments. Whether in socialized health care systems with potentially longer wait times and reduced access to cutting edge interventions, or in private health care systems with lack of universal health care, these choices are made invisibly by policies that limit services. But through collectively intelligent policies that optimize interventions across all outcomes, while algorithmically finding all possible chains of cooperation by which value might be created, these limits to services can be radically reduced over what is possible for either socialized health care or private health care today without GCI.

In GCI based health services, individuals would be free to select the health care that fits their political views. Those with left leaning political views would be free to choose socialized health care, and those with right leaning political views would be free to choose private health care. In both instances, case studies suggest GCI could reliably scale the value of cooperation within the sub-group choosing that option until that value allowed access to significantly more affordable health care, or significantly improved health care at the same cost, than any socialized medical system or private medical insurance is capable of today without GCI (Williams, 2020f).

A given sub-group in the collective might decide on a given set of health care expenditure policies. Whether such services are supported through taxes (national health care schemes) or supported by individuals directly (private health care schemes) would make little difference. They would still be customized to the individual according to their choices, through their choice of sub-group. These policies might be dependent on whatever factors the sub-group chooses, whether varying services based on factors such as eating a certain diet, exercising regularly, or avoiding substances that might affect their mental and physical health. In which case a GCI based simulation might constantly update the collective understanding of what an optimally healthy lifestyle is. Or these policies might be dependent on ignoring all those factors. Individuals might live whatever lifestyle or take whatever risky actions they would like, but in doing so they might incur whatever costs are agreed upon within the sub-group, such as increasing the deductible debited from their taxes (in national health care schemes) or from them directly (in private health care schemes) should they make any health care claims. As long as the risks and potential liabilities are balanced with the 
budget, then claims minus deductibles balance with the funds allocated to insure against or pay for the claims, and the system providing the services is balanced. Of course any aspect of such a system might be varied by the agreement of the sub-group. Individuals in one sub-group might agree the group should pay for 50

Each person will be able to join with a group of like-minded people, and therefore will be able to pay for other people to enjoy the services they would like others to pay for them to have, whether or not they are capable of paying at any given moment. Some costs will vary with time in a predictable way as well, so a group might include people who expect to enjoy services in the future that they can't utilize at the moment. For example, those people who would like to have children at some time in the future, might group themselves into the same insurance pool with people who have young children and those who wanted children but were never successful in having them or whose children are now adults.

What to do when one demographic consistently makes bad choices that lead to significantly worse well-being than another demographic, even when adjusted for inequalities? Or when one demographic allows another demographic to fall into deep disparity without acting? The answer is that since there are a potentially unlimited number of chains of cooperation by which outcomes might be increased, there are a potentially unlimited number of interventions by which individuals in that group can be incentivized towards choices that increase their well-being, and discouraged from choices that harm their well-being. And similarly there are a potentially unlimited number of interventions by which neighboring groups might be incentivized by the larger community to encourage disadvantaged communities towards positive choices and to discourage them from negative ones.

In the case of service policies that some communities find offensive and don't want other communities to have, but other communities believe are their right, the options are to ignore the transgressions of the other community, or to come into conflict with the other community. GCI can enable a continuum of coordinated actions from no action, to organized boycott, to coordinating physical intervention into other communities. Again, if two communities are in conflict, GCI must also engage the larger set of communities in executing policies that ensure both of those communities minimize the repercussions of that conflict on the larger community. GCI simply assists in choosing and optimum action. And for whatever action is chosen, GCI assists in optimizing outcomes of its executing.

GCI is an adaptive problem-solving system created to have dynamical stability so that it can sustainably explore the space of problems that can be defined related to maintaining that stability as well as to sustainably explore the solution space to those problems. Problems can be defined in terms of monopole or dipole properties, where those properties define outcomes. In the case of monopole properties, adaptive problem-solving involves a process of choosing the intervention that maximizes the property (outcome). And choosing the set of interventions that result in the optimal values of the set of properties (outcomes). In the case of dipole properties, adaptive problem-solving involves a process of choosing the intervention that balances the property. In the case of services, any number of monopole and/or dipole properties might be defined to characterize the service. Services are optimized by using GCI to apply adaptive problem-solving to the monopole and dipole properties of the service. In addition to monopole and dipole properties, higher order poles are theoretically possible, but might obey unstable dynamics that can't readily be used as a mechanism for optimizing services. One potential dipole property is payment timing. At a given budget, GCI adaptive problem-solving algorithms must create a balance between "pay before" methods such as payment through insurance fees for private services or tax revenue for public ones, and "pay after" methods such as deductibles for private services or tax credits for public ones. Another potential dipole property of services is the size of a chain of cooperation. At a given budget, GCI adaptive problem-solving 
algorithms must create a balance between the size of cooperation or the value of that cooperation, and constraints resulting from having to accommodate that number of cooperating entities. Through defining these properties as patterns of problems, and by defining patterns of solutions to these problems, a GCI can be used to significantly improve all government services by reusing these problem definitions and solution where they apply and where they have the potential for such improvement.

\section{IMPLICATIONS OF DECENTRALIZING COLLECTIVE DECISION-MAKING ON BRIDGING THE LEFT - RIGHT POLITICAL DIVIDE}

GCI aims to apply a methodical approach to decision-making in all topics. The very nature of the intuitive reasoning proposed to drive leftist cognitive bias likely leads to a tendency to reject any such efforts for topics that individuals on the left might believe are too complex to be reduced to reasoning that might come up with the "wrong" answers. However, using HCFM enables GCI to determine which outcomes maximize collective fitness (well-being). GCI then enables both the left and the right to objectively predict and measure optimal collective outcomes. Both sides can agree on this common desired goal even where agreeing on the path towards that goal is not reliably possible. Despite setting well-being as a universal goal, GCI doesn't make any assumptions about what type of reasoning will maximize collective outcomes. With GCI, truth is not defined by ideology. Truth is what works to achieve the best outcomes. A system (GCI) capable of finding truth must be capable of exploring the truth of all options, and must be capable of considering orders of magnitude more of those options so that none can systematically be excluded. A leftist cognitive bias might tend to conclude that any individual with "incorrect" reasoning should be prevented from following that reasoning for the greater good. A right leaning cognitive bias might fail to recognize that certain conclusions cannot be accepted regardless of the reasoning. Reasoning identifying what is in the interest of individuals, and therefore in the interest of the collective greater good, does not reliably convince those on the left where not in line with the left's conclusions, and cannot reliably prevail. In order to lower the left's resistance to other thought processes, and in order to lower the right's resistance to programs that restrict individual freedoms in the name of the collective good, GCI restricts any potential harm resulting from conservative policies to being inflicted on conservatives, and restricts any potential harm resulting from liberal policies to being inflicted on liberals. At the same time, GCI explores all these possible paths through conceptual space.

As a collective intervention, every service provided by the system of governance has inputs and their costs, as well as outputs and their benefits. In GCI based services, the governing entity (the system of governance executing) the intervention can take any measures the GCI makes available to increase the probability of achieving those benefits, to increase the magnitude of those benefits, and to make those benefits sustainably achievable. Collective interventions are achieved through collective activities executed by a number of roles. Some of these roles include choosing which activity to execute. Where a single process is collectively executed, and therefore involves multiple roles, the implications of being able to categorize all activities involved in the process as being defined by one context of execution or another, and the implications in being able to evaluate the context(s) in which executing those activities from a left or right leaning political bias is optimal, is that doing so creates the opportunity to use algorithms to more reliably optimize outcomes across all roles.

Optimizing collective outcomes across an entire service might be achieved by using GCI to orchestrate the process of selecting optimal options for each choice made by each role. For example, a department of agriculture might provide services to significantly improve agricultural livelihoods. In the case of the proposed Agricultural livelihoods program that comprises phase I of the proposed Collective Intelligence 
based Program to Accelerate Achievement of the Sustainable Development Goals (CIPAA-SDGs), a GCI based Social Impact Marketplace platform is intended to orchestrate the process of selecting farmers, providers of agricultural inputs, consumers of agricultural outputs, and other services providers, in deploying agricultural value chains that leverage collectively intelligent cooperation to gain the capacity to significantly increase collective outcomes (Williams, 2020d).

The implications of being able to reliably converge on optimal collective outcomes, while also being able to allow individuals to act according to what their individual cognitive biases suggest will optimize collective outcomes, is that a tremendous amount of time and resources are spent in right vs left campaigning, only to result in policies driven from either the left or from the right that conflict with each other in a way that is irreconcilable. In a GCI based approach, all governance policies and services anywhere, whether left leaning or right leaning, contribute to the exponential improvement of governance policies and services everywhere. While through the cognitive blockchain a reserve cryptocurrency with the capacity to reliably fund such such services can be deployed (Williams, 2020c).

\section{ELIMINATE ALL BIAS? OR INCLUDE ALL BIASES?}

Fitness in achieving a function is truth. Cognitive biases might exist at least to some degree because they are true within the context in which they exist. If an individual's context is not knowing which snakes are poisonous and which snakes are not, that individual will likely have a cognitive bias towards being terrified of snakes. That bias will be true because in that context snakes are truly terrifying. If an individual's context is being a snake scientist, they will likely not be terrified of snakes. That bias will also be true because in that context even poisonous snakes have predictable behaviors that allow the individual to interact with them without fear. Any given cognitive bias, or any type of thinking might be "true" or in other words "most fit" in a specific context. The goal of GCI is to improve decision making by collectively gaining the capacity to determine what that context is. One in a million times a conspiracy theorist will be dead right. And without them the conspiracy will never be discovered. One in a million times someone like Einstein will come up with a groundbreaking theory, potentially without any experimental data whatsoever. Should the possibility of those people be eliminated? It might be true that much of the innovation that moves the world forward happens with people who operate at the margin of some bias or another (i.e. they are extraordinarily biased towards a certain approach). A GCI might optimize collective outcomes through either having no bias ... or having every bias in the right amount at the right time. A functional modeling approach has the potential to determine truth (what functions to be true) more unambiguously than possible today. For example, in medicine, a functional model for the human system is currently being elaborated. In that functional model a sickness might be a well-defined pattern in that functional state space. Whether one uses homeopathy, western medicine, or traditional Chinese herbs, the impact of those treatment should be more clear than currently achievable through mere written case histories which might provide incomplete information from the perspective of any given treatment. For example, the Chinese treatment might depend on one's "Qi", data the western case history wouldn't catch. Functional modeling of the human system provides a way for medicine to catch all data. Functional modeling in every other area is meant to do the same rather than restricting data to the left or right narrative. The point is not just to develop a map for familiar territory, but to develop a compass for unfamiliar ground ... not just to learn which facts are true or not true within one's limited context, but instead to learn what the truth functions to achieve so that one can recognize truth in the unfamiliar facts of any context. 


\section{CONCLUSIONS}

Biases might increase problem-solving fitness in certain contexts given the limit to individual problemsolving ability, but no ideological bias (communism, socialism, capitalism, or other "ism"), no political bias (right, left, or centrist), no cognitive bias (rational methodical reasoning, or intuitive reasoning), nor any other bias of any other kind can increase problem-solving fitness as much as using GCI to exponentially increase general problem-solving ability and thereby using it to remove the current limits to problemsolving ability and to remove the current limits to the ability of populations to achieve collective well-being. Furthermore, the argument as to which bias is "better" is unresolvable between groups that each have one of these different biases, and therefore can't be resolved without conflict to eliminate those who disagree, or at least conflict to eliminate their ability to dissent. While this conflict might be satisfying to the victors, both biases have likely evolved because they each have deeply useful functions. Believing that question can be resolved, rather than having an understanding that even in the context in which a given bias is optimally fit, that bias is likely to be exponentially less fit at optimizing collective well-being than transitioning to a GCI, and therefore is not the end goal, by definition implies one has not developed sufficient understanding of the source of such biases to resolve the differences between them. A more productive solution would be implementing a GCI in order to maximize use of both of them.

Unbiased reasoning in the domain of sensitive subjects such as politics does not appear to be possible within the space of human cognition. The decision to apply the semblance of rational methodical reasoning to topics in which some conclusions are too offensive to be reached is a bias. The decision to apply intuitive reasoning to topics in which inconvenient conclusions are too impactful to be ignored is a bias. However there is great hope in focusing not on either bias, but on maximizing collective well-being through GCI. Both those with left or right leaning biases can agree that maximizing human well-being is a common goal. Combined with GCI, this common goal can potentially sweep away divisions. The application of GCI to politics is highly complex and potentially not for broad consumption. But the end conclusion is much simpler. Different reasoning must to be provided to justify GCI based services for the left from the reasoning used to justify such services to the right, because neither can reliably empathize with arguments from the other perspective. For the left, GCI enables services to be more inclusive while significantly improving collective outcomes over those that are possible today without GCI. For those on the right, GCI ensures individuals are free to make the choices that are essential to ensure individual well-being, and therefore are required to ensure the collective well-being of the group. While ensuring these individual freedom, GCI significantly improves collective outcomes over those that are possible today without GCI.

\section{CONFLICT OF INTEREST STATEMENT}

The authors declare that the research was conducted in the absence of any commercial or financial relationships that could be construed as a potential conflict of interest.

\section{AUTHOR CONTRIBUTIONS}

All contributions to this article were made by the author.

\section{FUNDING}

This work was conducted independently without external funding.

\section{ACKNOWLEDGMENTS}

Many thanks to Julian Talev for endless discussions on the topic of GCI. And many thanks to Juhi Sonrexa for debate regarding this specific paper. 


\section{REFERENCES}

Baron, J. and Jost, J. T. (2019). False equivalence: Are liberals and conservatives in the united states equally biased? Perspectives on Psychological Science 14, 292-303. doi:10.1177/1745691618788876. PMID: 30836901

Bernhardt, D., Krasa, S., and Polborn, M. (2008). Political polarization and the electoral effects of media bias. Journal of Public Economics 92, 1092 - 1104. doi:https://doi.org/10.1016/j.jpubeco.2008.01.006

Bowen, R., Dmitriev, D., and Galperti, S. (2020). Learning from shared news: When abundant information leads to belief polarization

Béland, D. and de Chantal, F. V. (2004). Fighting "big government": Frames, federalism, and social policy reform in the united states. The Canadian Journal of Sociology / Cahiers canadiens de sociologie 29, 241-264

Groseclose, T. (2012). Left turn: How liberal media bias distorts the American mind. Perspectives on Politics 10, 775-779. doi:DOI:10.1017/S1537592712001387

Kidder, J. L. (2018). Civil and uncivil places: The moral geography of college republicans. American Journal of Cultural Sociology 6, 161-188. doi:10.1057/s41290-016-0023-5

Lewis, S. C. (2020). Lack of trust in the news media, institutional weakness, and relational journalism as a potential way forward. Journalism 21, 345-348. doi:10.1177/1464884918807597

LUTTBEG, N. R. and GANT, M. M. (1985). The Failure of Liberal/Conservative Ideology as a Cognitive Structure. Public Opinion Quarterly 49, 80-93. doi:10.1086/268902

Malone, T. W. (2018). Superminds: The surprising power of people and computers thinking together. (Little, Brown Spark)

Marshall, S. E. (1996). Marilyn vs. hillary:. Women \& Politics 16, 55-75. doi:10.1300/J014v16n01 \_03

M.D., E. S. (1981). Empathy: A mode of analytic listening. Psychoanalytic Inquiry 1, 357-392. doi:10. 1080/07351698109533410

Morgan, S. (2018). Fake news, disinformation, manipulation and online tactics to undermine democracy. Journal of Cyber Policy 3, 39-43. doi:10.1080/23738871.2018.1462395

Rapaport, W. J. (1982). Unsolvable problems and philosophical progress. American Philosophical Quarterly 19, 289-298

Saka, Y. and Cohen-Louck, K. (2014a). From demonization to identification: How parents who lost children in terrorist attacks perceive the attacker. Journal of Loss and Trauma 19, 137-154. doi:10.1080/ 15325024.2012.743323

Saka, Y. and Cohen-Louck, K. (2014b). From demonization to identification: How parents who lost children in terrorist attacks perceive the attacker. Journal of Loss and Trauma 19, 137-154. doi:10.1080/ 15325024.2012 .743323

Sipser, M. (1996). Introduction to the theory of computation. SIGACT News 27, 27-29. doi:10.1145/ 230514.571645

Washburn, A. N. and Skitka, L. J. (2018). Science denial across the political divide: Liberals and conservatives are similarly motivated to deny attitude-inconsistent science. Social Psychological and Personality Science 9, 972-980. doi:10.1177/1948550617731500

Williams, A. E. (2019). The relationship between collective intelligence and one model of general collective intelligence. In Computational Collective Intelligence, eds. N. T. Nguyen, R. Chbeir, E. Exposito, P. Aniorté, and B. Trawiński (Cham: Springer International Publishing), 589-600

Williams, A. E. (2020a). Research Square doi:10.21203/rs.3.rs-18022/v1

Williams, A. E. (2020b). Assessing the potential impact of general collective intelligence doi:10.31730/osf. io/e5usx 
Williams, A. E. (2020c). The cognitive blockchain and the internet of value

Williams, A. E. (2020d). The collective intelligence based program to accelerate achievement of the sustainable development goals as a case study for collectively intelligent program design

Williams, A. E. (2020e). General collective intelligence and the constraints to group decision-making doi:10.31234/osf.io/6gten

Williams, A. E. (2020f). General collective intelligence as a paradigm in healthcare

Williams, A. E. (2020g). The global response to covid-19 as an example of a one-sided problem definition in the absence of general collective intelligence doi:10.31234/osf.io/emgxc

Williams, A. E. (2020h). A human-centric functional modeling framework for defining and comparing models of consciousness and cognition doi:10.31234/osf.io/94gw3

Williams, A. E. (2020i). Human intelligence and general collective intelligence as phase changes in animal intelligence doi:10.31234/osf.io/dr8qn

Williams, A. E. (2020j). A model for artificial general intelligence. In Artificial General Intelligence, eds. B. Goertzel, A. I. Panov, A. Potapov, and R. Yampolskiy (Cham: Springer International Publishing), 357-369

Williams, A. E. (2020k). A model for general collective intelligence doi:10.31730/osf.io/6u984

Williams, A. E. (20201). Use of human-centric functional modeling to maximize convergence in integrative research doi:10.31730/osf.io/jv6h8

Woolley, A. W., Chabris, C. F., Pentland, A., Hashmi, N., and Malone, T. W. (2010). Evidence for a collective intelligence factor in the performance of human groups. Science 330, 686-688. doi:10.1126/ science. 1193147

Yankelovich, D. (2015). Wicked problems workable solutions: Lessons from a public life. (London: Rowman Littlefield.)

Zhuk, D., Tretiakov, A., Gordeichuk, A., and Puchkovskaia, A. (2018). Methods to identify fake news in social media using artificial intelligence technologies. In Digital Transformation and Global Society, eds. D. A. Alexandrov, A. V. Boukhanovsky, A. V. Chugunov, Y. Kabanov, and O. Koltsova (Cham: Springer International Publishing), 446-454 